\title{
Terroranschläge in Frankreich seit 1995: ein postkoloniales Drama
}

Die Terroranschläge im Namen von Al-Qaida im Januar 2015 in Frankreich können als ein postkoloniales Drama gedeutet werden: ein Drama zunächst im Sinne des Schocks, den die Gewalt und der Schmerz ausgelöst haben. Aber auch aus einem analytischen Blickwinkel - nämlich im Gegensatz zum Begriff der Tragödie. Während sich in der Tragödie auf der Grundlage göttlicher, ideologischer oder materialistischer Mechanismen ein Determinismus entfaltet, wird das Drama als Ergebnis eines Bündels kontingenter Beziehungen, Handlungen und Erfahrungen aufgefasst, die eigentlich nicht zu diesem Ausgang hätten führen sollen. Eine Deutung als Drama rückt die, relationale Konstruktion' in den Blickpunkt: Hier steht nicht mehr die Notwendigkeit des Ereignisses, sondern die Bedingungen seiner Ermöglichung im Zentrum der Frage - und dies sowohl auf der historischen und geopolitischen als auch auf der subjektiv-individuellen Ebene. Das Konzept ,postkolonial' darf nicht mit der Idee einer kolonialen Kontinuität oder, was auf dasselbe hinausläuft, mit dem Konzept des ,Neokolonialen“ verwechselt werden. Ein koloniales Verhältnis setzt voraus, dass es Kolonialisierte und Kolonisierende gibt. Heute bestehen solche Verhältnisse nicht mehr - auch nicht zwischen Israel und Palästina, wo es sich eher um eine strafende Segregation handelt (durch die Eingrenzung in Gaza und die partielle Annexion und Besetzung im Westjordanland). Das Postkoloniale soll vielmehr als die Gesamtheit aller gegenwärtigen Machtverhältnisse verstanden werden, die aus der Kolonialisierung und der Entkolonialisierung hervorgehen (Hall 2007).

Für die hier besprochene Fragestellung sind die Beziehungen zwischen den westlichen Staaten und den Staaten des Nahen Ostens und des Maghreb von besonderer Bedeutung. Allgemeiner betrachtet ist die postkoloniale Beziehung Bestandteil der Kosmopolitisierung der Welt bzw. der „glokalisierten“, wechselseitigen Abhängigkeitsverhältnisse der Zweiten Moderne (Beck 2004): Es gibt in der Welt der Zweiten Moderne kein ,Außen' mehr, das sich den Rückwirkungen der globalisierten Handlungen und sozialen Beziehungen entziehen könnte (Beck 2002). Davon ausgehend ist es meines Erachtens möglich, sowohl die 1995 von der GIA (Groupe Islamique Armé[1]) angeordneten Anschläge in Frankreich als auch die mit Al-Qaida in Zusammenhang stehenden Anschläge 
von 2012 und 2015 als Ergebnis des dramatischen Aufeinandertreffens zweier postkolonialer Handlungslogiken zu deuten. Dies sind einerseits Logiken, die zur Bildung eines revolutionären Dschihadismus im Nahen Osten und im Maghreb führen, und andererseits Logiken, die zu den sozialen und subjektiven ,Karrieren' (im Sinne von Becker 1981) einer ultrakleinen Minderheit französischer Nachfahren postkolonialer Migrant_innen führen, die zu Dschihadist_innen in ihrem eigenen Land und gegen ihr eigenes Land geworden sind.

\section{Die postkoloniale Fabrik des revolutionären Dschihadismus in Gesellschaften mit muslimischer Mehrheit}

Die Hauptkonsequenzen der Kolonialisierung und Entkolonialisierung der arabischen Gesellschaften sind die Errichtung - im politikwissenschaftlichen Sinne - modernisierender Regimes autoritärer, nationalistischer, plutokratischer und/oder oligarchischer Prägung und Allianzen mit dem Westen, der so seine Energieinteressen verteidigt. Wie die ehemaligen Kolonialherren üben diese autoritären Regime ihre Macht aus, indem sie einerseits die Herausbildung einer Zivilgesellschaft verhindern, weil sie die Demokratisierungsbestrebungen der Mittelschicht als Bedrohung erachten, und andererseits jede Form des politischen Islams, das einzige potentielle Sprachrohr eines Volksprotests, gewaltsam unterdrücken. Somit lassen sie dem politischen Islam keinen Raum für eine modernisierende und demokratische Entwicklung. Nach dem Scheitern der arabischen Kriege gegen Israel haben diese Regimes de facto die palästinensische Sache aufgegeben und somit genau wie die westlichen Staaten die Politik der vollendeten Tatsachen des israelischen Staats stillschweigend gebilligt.

In der schiitischen Welt kann die islamische Revolution 1979 im Iran als erfolgreiches Ergebnis des politischen und ideologischen Widerstands in der schiitischen Welt gegen diese Allianz von autoritären Regimen des Nahen Ostens und dem Westen gedeutet werden. In der sunnitischen Welt hat die revolutionäre und militarisierte Bewegung des neoislamischen Dschihadismus, welche von Al-Qaida bereits 1987 gegründet wurde, sowohl die modernisierenden, autoritären Regimes als auch deren westliche (Saudi Arabien und die USA an erster Stelle, aber auch Ägypten) oder schiitische Alliierten (Syrien) als Gegner. In beiden Fällen werden die Motive dieser Bewegungen von einem tiefliegenden arabischen und muslimischen Ressentiment genährt, welches sich auf einen antisemitistischen und antiisraelischen Antizionismus stützt, der durch jede Verschärfung der Repression durch Israel gestärkt wird. Durch die Kombination von kurzfristigen (Sturz der lokalen Regierungen) und langfristigen Zielen (Schwächung der westlichen Unterstützung) hat sich dieser revolutionäre Dschihadismus in einen, asymmetrischen Krieg' gestürzt: einen von den antikolonialen Befreiungskriegen übernommenen Krieg des Schwachen gegen den Starken (Chaliand 2008) - insbesondere in Algerien gegen die Franzosen und in Afghanistan gegen die Sowjets. Er wird zu einem transnationalen ,Krieg der Zivilisationen' gemacht, der von der Idee einer historischen Revanche im Maßstab von Jahrtausenden durch die Anhänger einer neoislamitische religiöse Ideologie angetrieben wird.

Symmetrisch dazu kann der größtenteils von jungen kosmopolitischen Menschen getragene ,Arabische Frühling“ von 2011 als die demokratische 
Version des Aufstands gegen die aus der Entkolonialisierung entstandene autoritäre postkoloniale Ordnung gedeutet werden. Nun, da die Offensive des Dschihadismus den postkolonialen Status quo bedroht, haben die USA, begleitet von den anderen westlichen Staaten, die Vorzeichen des ,Kriegs der Zivilisationen' akzeptiert und ihn in einen,Krieg gegen den Terror umbenannt - eine Politik, die von den selbst oft terroristischen kolonialen Techniken des Gegenterrorismus und Gegenaufstandes herrührt (Scahill 2013). Dies hatte zwei Effekte: Einerseits wurden die autoritären Regime in ihrem Kampf gegen den politischen Islam gestärkt, auch wenn dabei die Zivilgesellschaft der ,Arabischen Frühlinge‘ beseitigt wird (wie in Ägypten). Andererseits wurde der absolute, Kriegszustand' in Afghanistan und im Irak wiederbelebt. Die Konsequenz ist ein militärisches, wirtschaftliches, ethno-religiöses und ideologisches Chaos, welches zahlreiche Menschenleben kostet und dadurch das begünstigt, was hätte bekämpft werden müssen - und dessen direktes Ergebnis die Entstehung des Islamistischen Staates ist (Shaw 2005).

Das politische Ziel der antikolonialen Kämpfe war die Abschaffung der kolonialen Asymmetrie zugunsten einer symmetrischen wechselseitigen Anerkennung. Der revolutionäre Dschihadismus und der ,Krieg gegen den Terror radikalisieren jedoch die Spannungen, indem sie der westlichen postkolonialen Asymmetrisierung (Butler 2010) eine reziproke dschihadistische Asymmetrisierung entgegensetzen. In beiden Fällen ist das Leben der ,Anderen - das der ,terroristischen“ Bevölkerung einerseits und das der westlichen Bevölkerung und ihrer Alliierten andererseits - wertlos in einem Krieg, der keine räumlichen, zeitlichen, rechtlichen und technischen Grenzen mehr hat. Diese Radikalisierung lässt sich auch im paradigmatischen Konflikt zwischen Israel und den Palästinensern beobachten: Dieser war zunächst lange Zeit ein klassischer nationalistischer, antikolonialistischer Kampf gegen den Einfluss des israelischen Staates in Gaza und im Westjordanland. Die UNO sollte dabei als Schiedsrichter fungieren. Der Konflikt gleitet nun aber ohne westliche Reaktion immer weiter in Richtung eines ,Kriegs der Zivilisationen“ ab. Er wird ausgetragen zwischen dem ethno-religiösen, rassistischen Extremismus in Israel einerseits - ein Extremismus, der das Antlitz einer Politik des ,geringsten Schadens' annimmt, bewaffnet durch das Recht und den technologischen Vorsprung (Weizman 2011) -, und dem ethno-religiösen, antisemitischen und antiwestlichen Extremismus in Palästina andererseits. Allgemein gesprochen - und ohne, dass es hier möglich wäre, einfache ,Lösungen“ für diese Verwerfungen zu erkennen - kann sehr wohl eine Koproduktion des revolutionären Dschihadismus beobachtet werden. Und zwar in der Art und Weise, wie die geopolitische postkoloniale Ordnung von den Gesellschaften des Nahen Ostens und denen der westlichen Gesellschaften definiert wird, wobei der Dschihadismus als eine der kosmopolitisierten Rückwirkungen der Beziehungen von Westen und Nahem Osten erscheint.[2]

\section{Soziale und subjektive Werdegänge junger (postmigrantischer), postkolonialer Franzosen und Französinnen, die zu Dschihadist_innen gewordenen sind}

Die Kehrseite dieses dramatischen Aufeinandertreffens ist, dass die französische Gesellschaft nicht nur die dschihadistischen Praktiken selbst 
hervorbringt, sondern auch die Bedingungen ihrer Entstehung. Tatsächlich werden kaum junge französische Nachfahren postkolonialer Migrant_innen zu Dschihadist_innen. Jene, die es werden, teilen eine ähnliche Erfahrung mit der französischen Gesellschaft, die hart mit den Jugendlichen aus armen Verhältnissen umgeht und die weniger die gesellschaftliche Integration als die soziale ,Desintegration “ fördert: die Konzentration der Armut in einzelnen Stadtvierteln; ein elitäres Schulsystem, das durch Scheitern selektiert; eine lang währende Prekarität beim Eintritt in den Arbeitsmarkt; sowie eine kulturelle und subjektive Ghettoisierung, die zum Auftreten von marginalisierten Subkulturen führt (Lapeyronnie 2008). Hinzu kommt für die Nachfahren postkolonialer Migrant_innen die Erfahrung, jederzeit zum Opfer von Rassismus, Stigmatisierung und Diskriminierung werden zu können - wenn sie das nicht bereits wurden. Während sie diese Erfahrungen als ein ,Problem' empfinden, das sie daran hindert, ein ,normales Leben` zu führen, vermitteln ihnen die meisten medialen und politischen Darstellungen, sie selbst seien das Problem, sei es nun als Nachfahren von Migrant_innen, als Muslime oder gar als Individuen (Dubet et al. 2013). Diese Erfahrung kann bei manchen zu einem tiefen Unterdrückungsgefühl führen, welches das verstärken kann, was François Dubet bereits in den $1980 e r$ Jahren als die ,Wut der Unterschicht ${ }^{6}$ beschrieb, die keine politische Unterstützung durch die alten Traditionen des Arbeiterwiderstandes mehr erfährt (Dubet 1987). Diese,Wut' kann entlang einer sozialen und subjektiven ,Karriere' aus Zwischenschritten, die umkehrbar sein können, zu einer Kultur des Ressentiments und zur narzisstischen Dekompensation führen - oft vor dem Hintergrund eines komplizierten familiären und emotionalen Lebens: Mangel an emotionaler Zuwendung, Gewalt, Aufenthalte in Pflegeheimen. Von dort eröffnen sich für einige zahlreiche Möglichkeiten zur Überschreitung der Normen von Leben und Tod je nach kultureller und politischer Verankerung der einzelnen ,Karriere‘ und den ,Vorteilen' bei der eigenen Subjektivierung (Khoroskhavar 2014). Dies kann sich äußern in unkontrollierbaren, wütenden, an Wahnsinn grenzenden Gewaltausbrüchen und/oder in Delinquenz und Kriminalität und/oder sektiererischem muslimischen Neofundamentalismus und/oder politischreligiösem neomuslimischen, antiwestlichen Radikalismus, verbunden mit einem mit Antizionismus verwechselten Antisemitismus.

Die Begegnung von Subjektivitäten, die meinen, ihren Tod besser gestalten zu können als ihr Leben, mit den Logiken des Dschihadismus ist nicht zwingend und bleibt extrem selten, denn es gibt viele gute Gründe für alle, die diese sozialen Erfahrungen teilen, kein Dschihadist zu werden und ein ,normales Leben' anzustreben. Dennoch stehen diese ,verfügbaren“ Subjektivitäten im Zentrum der Strategien des Dschihadismus, der schon seit langem klassische und neue Methoden der Anwerbung, Rekrutierung, Ausbildung und Unterstützung für terroristische - am häufigsten selbstmörderische - Aktionen beherrscht. Diese Praxis ist seit der terroristischen Anwerbung von Khaled Khelkal durch die GIA bekannt, deren Ziel es war, während des algerischen Bürgerkriegs der 1990er Jahre[3] Druck auf Frankreich auszuüben und reicht bis zur Anwerbung von Mohamed Merah, Amedy Coulibaly und der Brüder Kouachi durch Untergruppen von Al-Qaida - ganz abgesehen von den nun noch hinzu kommenden Effekten der ,vernetzten“ Anwerbungsmethoden des Islamischen Staates. 


\section{Von einer Koproduktion zur anderen}

Es wird nun deutlich, dass der ,Krieg gegen den Terror ${ }^{\star}$ und die quasi-religiöse Verherrlichung der ,Werte der Republik' als Antwort auf den ,Krieg der Zivilisationen' weniger Lösungen als Teil des Problems Dschihadismus darstellen und seine Attraktivität weiter erhöhen. Diese Erkenntnis lässt mehrere Schlüsse zu: Einerseits sind alle guten Gründe‘ dafür vereint, dass der Dschihadismus existiert und weiterhin rekrutiert. Andererseits bleibt dieses Engagement für den tödlichen Dschihadismus minoritär, so dass bei jedem Zwischenschritt dieser Logiken und dieser ,Karrieren“ der Schritt in den Dschihadismus verhindert werden könnte: durch die Koproduktion einer weniger ungerechten postkolonialen Ordnung. Hierfür sind Politiken der demokratischen Transition und der Umverteilung denkbar, wie etwa in Tunesien. Selbst in Palästina, Nigeria, Mali und anderen Ländern würden sie zur Entstehung einer Zivilgesellschaft führen, die die dschihadistische „Lösung'verhindern könnte. Dies wäre allerdings ein überaus umfangreiches Reformprogramm, was nachvollziehbar werden lässt, weshalb sich meist die ,Lösung‘ in Form des ,Kriegs gegen den Terror' durchsetzt. Andererseits muss an der Verhinderung dieser dschihadistischen ,Karrieren' gearbeitet werden, und zwar durch konsequente Politiken gegen Stigmatisierungs-, Diskriminierungs- und Ausgrenzungsmechanismen in Schule und Stadt. Ein solches Programm wäre durch und durch reformistisch. Es würde sich vom dem republikanischen Begriff der,Brüderlichkeit inspirieren lassen und sich den Logiken der heute in Frankreich vorherrschenden „Bevorzugung sozialer Ungleichheit“(Dubet 2014) entgegenstellen. Und es sollte umgesetzt werden, bevor der größte Kollateralschaden - wenn nicht sogar das strategische Ziel - des dschihadistischen Terrorismus in Frankreich und überall im Westen eintritt: der Import des ,Krieges der Zivilisationen“ in die französische Gesellschaft durch die gemeinsame Aktion der dschihadistischen Radikalen, der rechtsradikalen Ethnonationalist_innen und der,republikanischen' Radikalen, die eine staatliche Gewalt fordern, deren Konsequenz der Niedergang der Zivilgesellschaft und der Demokratie wäre.

Übersetzung aus dem Französischen ins Deutsche:

Mélina Germes, Philippe Kersting, Nina Schuster und Andreas Tijé-Dra

\section{Endnoten}

[1] GIA, Groupe Islamique Armé (dt.: bewaffnete islamische Gruppe) bezeichnet die Gruppen, die nach der Annullierung der ersten freien Parlamentswahlen in Algerien 1992, bei denen die Islamische Heilsfront gewann, einen Bürgerkrieg ausgelöst haben (Anmerkung der Übersetzer_innen).

[2] Zum Begriff der Koproduktion von Gewalt und Unsicherheit durch die Akteure die sie bekämpfen sollten, siehe Macé 1997.

[3] Einige Jahre vor den Anschlägen Khaled Kelkals 1995 interviewte der deutsche Soziologe Dietmar Loch ihn in Vaulx-en-Velin im Rahmen einer Doktorarbeit über die Integration junger Franko-Maghrebiner. Dieses Interview wurde von der Tageszeitung Le Monde 1995 veröffentlicht, um den sozialen und subjektiven Werdegang dieses ersten französischen Dschihadisten, der gegen Frankreich handelte, zu veranschaulichen. 


\section{Autor_innen}

Éric Macé ist Soziologe und Politikwissenschaftler.

eric.mace@u-bordeaux.fr

\section{Literatur}

Beck, Ulrich (2002): The terrorist threat: World risk society revisited. In: Theory, Culture \& Society. 19: 39-55.

Beck, Ulrich (2004): Qu'est-ce que le cosmopolitisme? Paris: Aubier.

Becker, Howard (1981): Außenseiter: zur Soziologie abweichenden Verhaltens Outsiders. Etudes de sociologie de la déviance. Frankfurt am Main : Fischer, 1981.

Butler, Judith (2010): Raster des Krieges: Warum wir nicht jedes Leid beklagen. Frankfurt am Main: Campus.

Chaliand, Gérard (2008): Les guerres irrégulières: XXe-XXIe siècle. Guérillas et terrorismes. Paris: Gallimard-Folio.

Dubet, François (1987): La galère: Jeunes en survie. Paris: Fayard.

Dubet, François (2014): La préférence pour l'inégalité. Comprendre la crise des solidarités. Paris: Seuil

Dubet, François; Cousin, Olivier; Macé, Eric; Rui, Sandrine (2013): Pourquoi moi? L'expérience des discriminations. Paris: Seuil.

Hall, Stuart (2007): Quand commence le ,postcolonial` ? Penser la limite. In: Identités et cultures. Politiques des Cultural Studies. Paris: Éditions Amsterdam.

Khosrokhavar, Farhad (2014): Radicalisation. Paris: Éditions de la MSH.

Lapeyronnie, Didier (2008): Ghetto urbain. Ségrégation, violence, pauvreté en France aujourd'hui. Paris: Robert Laffont.

Macé, Éric (1997): Service public et banlieues populaires : une coproduction de l'insécurité. Le cas du réseau bus de la RATP. Sociologie du Travail, $\mathrm{n}^{\circ} 4$ (473-498)

Scahill, Jeremy (2013): Schmutzige Kriege: Amerikas geheime Kommandoaktionen. München: Kunstmann.

Shaw, Martin (2005): The New Western Way of War. Risk-Transfer and its Crisis in Iraq. Cambridge: Polity Press.

Weizman, Eyal (2011), The Least of All Possible Evils. Humanitarian Violence from Arendt to Gaza. London: Verso. 